\title{
Survey and Analysis of Wushu Sanda Development Status Based on SPSS Statistics
}

\author{
Huabei Jiang, Songshan Zhou, Zhiming Qin \\ Hebei Institute of Physical Education \\ Shijiazhuang 050041, Hebei, China \\ tiyuxi@qq.com
}

\begin{abstract}
By means of the literature data, interview, questionnaire, mathematical statistics and logic analysis method, this paper gets a comprehensive understanding of the health clubs in Nanjing downtown, including the venue status analysis, operation mode analysis, charge situation analysis, situation analysis of the Wushu Sanda practitioners and coaches in the Wushu Sanda clubs. Studies have shown that Wushu Sanda development of the fitness clubs is in an upward trend overall and has a great potential for development. However, there are also some factors that constraint Wushu Sanda practitioner to participate in the practice of club Wushu Sanda, such as the club has a less number of experienced and professional Sanda coaches and there is still much room for improvement of the quality of some of the coaches. Besides, the Wushu Sanda clubs possess a single business model, which mainly is small-scale operation. And the Sanda training conditions provided by the clubs is relatively poor, so it needs to further increase capital investment in hardware conditions to improve it significantly. Based on these studies, in the end, this article puts forward meaningful recommendations for the further development of Wushu Sanda in Nanjing fitness clubs, in order to better serve the public.
\end{abstract}

Keywords—Statistics; SPSS; Club; Status analysis

\section{INTRODUCTION}

With the improvement of the economic level and the implementation of the "National Fitness Program", China's sports career has ushered in a good development opportunity. As a part of the martial arts soul, Sanda, on the basis of the martial arts, refines the most simple and direct moves. In accordance with certain rules, Sanda is a competitive fighting game project in the form of kicking, throwing, hitting and seizing. In order to promote the rapid development of Sanda and to address the growing demand for Sanda, Sanda exist not only in the provincial and municipal sports schools and martial arts schools, it is also flourishing in the forms of the clubs or martial arts school in small, medium and large cities, which actively promotes the prosperity and development of Wushu Sanda projects in China.

Many studies have shown that the sports industry in the broad environment of China's market economy is gradually developing and expanding, and Sanda Club, as a rising star of sports industry is gradually rising. Sanda Clubs in Beijing, Shanghai, have begun to take shape; Qingdao and Jinan of Shandong also have a professional
Sanda club; for Chengdu and Xi'an in the western, the Sanda club's operations are better. Jiangsu Province is a sports province and Nanjing carried out earlier in Sanda. The survey shows that the development of Wushu Sanda in Nanjing is mainly dependent on the major health clubs, which are located in Nanjing downtown. The main mode of operation for these clubs is small scale and independent operation. After several years of development, these clubs have accumulated a certain market space and mass base.

On the whole, in recent years, the Wushu Sanda in fitness clubs experiences certain development, but there are still a lot of problems. How to actively and effectively develop the Wushu Sanda in fitness clubs in Nanjing has become an urgent issue, which is also related to the improvement of the overall level of the Wushu Sanda. However, through a survey of the literature and access to the club staffs, deep discussion of this problem has not yet been found. Therefore, on the basis of in-depth survey research, this study will put forward corresponding proposals for the work through the analysis of existing problems, aiming at to provide a valuable reference for the further development of the Wushu Sanda in fitness clubs in Nanjing.

\section{RESEARCH OBJECTS AND METHODS}

In this study, the survey are mainly carried out aiming at the curators, coaches and trainees form six fitness clubs in Nanjing (Nanjing Surreal Fitness Center, Nanjing Jiangning District National Fitness Center, Nanjing Suntech Fight Club, Oriental Fighter Fitness Club, Wutai Mountain Fitness Center, Nanjing Gulin Fitness Center).

Research methods

(1) Literature method: through access to the Library of Nanjing Institute of Physical Education, China academic periodical net, China Info, as well as Chinese periodicals library (Chongqing VIP) and other information resources, this study conducts survey research on the details of Wushu Sanda. Meanwhile, the authors also read the Wushu Sanda literature (such as martial arts and taekwondo). Collation and analysis of the information in the above literature lays a theoretical foundation for the writing of this article.

(2)Questionnaire: the designing work of the questionnaire is processes after accessing to a lot of the literature related to this topic. Combining with the research purpose, meaning and content of this paper, the authors preliminarily design the questionnaire. The questionnaires 
in this paper are designed for all the Wushu Sanda Club members. The questions are easy to understand and convenient to fill out. Modify and supplement the questionnaire by referring to materials and reach the final draft after improvement.

The issuance and recycling of questionnaires: The implementation of the issuance of the questionnaires to 6 Sanda clubs in Nanjing is conducted by the authors personally. The questionnaires are filled out and recycled on the spot. According to statistics, a total of 121 questionnaires are handed out and 121 copies recycled. Wherein, the number of questionnaires sent to the persons in charge is 6 and the 6 questionnaires are all recycled. Recovery rate and efficiency rate are both $100 \%$. The number of questionnaires handed to the coaches is 15 , with $100 \%$ of recovery and efficient rate. The number of questionnaire sent to the participants is 100 parts and the 100 questionnaires are all returned with 100valid questionnaires. So the recovery rate and efficiency rate are both $100 \%$.

The questionnaire's validity testing: by repeat testing methods, 15 days after the first issuance of questionnaires, randomly select 20 trainees from the Wushu Sanda clubs in Nanjing City and once again do the issuance of questionnaires to investigate the validity of the questionnaire. The test results show the credibility of this questionnaire.

(3) Mathematical statistics: According to the survey methods and statistical principles, the data obtained from the survey are for conventional mathematical statistics. And statistical analysis using SPSS and Excel software on the contents of the questionnaire is conducted, which includes the site area of the club, mode of operation, the charges situation, the practitioner related information and the coaches related information.

(4) Logic analysis: Based on the principles and methods of logic, including induction, analogy, interpretation, and integration, etc, logic analysis methods, this study conducts comprehensive analysis and discussion of a variety of information. Emphasizing on identifying the key reasons for the problems revealed, the guiding ideology is that when multiple variable factors are related to the generation of the problem, strive to find out one of the most critical factors and demonstrate the relevant conclusions and make recommendations accordingly.

\section{SURVEY RESULTS AND ANALYSIS}

\section{A. Status analysis of the Wushu Sanda Clubs}

By means of interviews and investigations, this study finds that the several Wushu Sanda Clubs are all in independent operating mode, and that the open mode jointed by enterprises or company has not yet appeared. In order to let more people learn Sanda and bring more convenience to the students, there is one Wushu Sanda club that is in a chain form of operation. Club locations are all near luxury cells, aiming at the high school students, college students and the relatively high levels of economic groups. It is found in the survey that current development of Taekwondo in Nanjing introduces the club league mode, forming a broad and relatively fixed mass base. By means of perfect competition system, more people participate in this sport and are fond of this sport, which expands and promotes the flourishing of Taekwondo in Nanjing region. At the same time, the organization of the club league enriches the club's sources of funds (such as ticket sales, advertising revenue, the league sponsorship revenue, etc.), which can also provide experience and reference for the further development of Sanda Clubs.

As the scale and training conditions of the several Sanda clubs are different, the fees are also different. As shown in Table 1 , the clubs involved in the survey all charge a monthly fee: the number of clubs with an average monthly fee of $300 Y$ is 4 and the number of clubs with an average monthly fee of $150 ¥$ is 2. Judging from these data, the charges of Sanda clubs in Nanjing are basic reasonable and there is no chaos phenomenon of the charges. The survey shows that the price difference is mainly influenced by the business scale and training conditions of the clubs. Four large-scale health clubs possess good training venues and related equipment and the professional quality of the coaches also is relatively high. As a result, these clubs charges as more than two times that of the other two smallscale clubs.

TABLE 1 TUITION SITUATION OF THE WUSHU SANDA CLUBS

\begin{tabular}{|c|c|c|}
\hline Tuition & Numbers & Percentage \\
\hline $150 ¥$ & 2 clubs & $33 \%$ \\
\hline $300 ¥$ & 4 clubs & $67 \%$ \\
\hline
\end{tabular}

\section{B. The current situation of Wushu Sanda practitioners}

(1) Analysis of Sanda information channels for Wushu Sanda practitioners. As can be seen from Table 2, the number of practitioners getting to know about Sanda through network and TV is 84 , accounting for $84 \%$ of the total number; the number of practitioners getting to know about Sanda through books, newspapers and periodicals is 10 , accounting for $10 \%$ of the total number; the number of practitioners getting to know about Sanda through friends is 6 , accounting for 6 of the total number; judging from these data, the main channel for people to get Sanda information are still mainly trough network and TV. The information retrieval channels are single, which affects the faster and better development of the Wushu Sanda, so it is necessary to continue to increase the publicity efforts.

TABLE 2 PERCENTAGE OF SANDA INFORMATION CHANNELS FOR THE WUSHU SANDA CLUB TRAINEES

\begin{tabular}{|c|c|c|}
\hline Channels & Number & Percentage \\
\hline Network and TV & 84 people & $84 \%$ \\
\hline Books, newspapers and periodicals & 10 people & $10 \%$ \\
\hline Through friends & 6 people & $6 \%$ \\
\hline
\end{tabular}


(2) Gender analysis of the Wushu Sanda practitioners. The number of male Sanda practitioners is 97, accounting for $97 \%$; and the number of female Sanda practitioners is 3 , accounting for $3 \%$. As can be seen from Figure 1, there are more males and less females, which may be related to the characteristics of Sanda. As Sanda is highly confrontational project and requirements of physical fitness and overall quality are relatively high, it may prevent females to participate in Sanda.

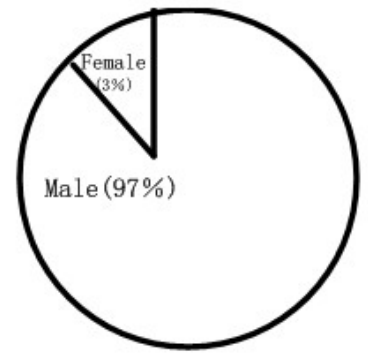

Fig. 1. Gender Structure of the Wushu Sanda Club Students

(3)Age structural analysis of the Wushu Sanda practitioners. As can be seen from Table 3, trainees of 1821-year-old is the most among the trainees, accounting for $50 \%$, followed by the 22-25-year-old trainees, demonstrating that it is mainly young people participating in Sanda clubs. Trainees of 14-17-year-old account for 7\% of the total number. If merely analyzing from the perspective of exercise physiology, the best stage for the development of speed, flexibility and explosiveness for boys and girls are respectively 10-14 years old and 10-12 years old. However, judging from the questionnaire data, the number of trainees at this stage is zero, which misses the best period for training and also is a key factor that results in the failure to select a good back-up talent. The number of people participating in the Sanda over 25 years old shows a downward trend, mainly because graduate students, working people and members of society account for the majority of this age group and they can only use their spare time to practice.

TABLE 3 Age STATISTICS OF THE WUSHU SANDA PRACTITIONERS

\begin{tabular}{|c|c|c|}
\hline Age & Number & Percentage \\
\hline $14-17$ years old & 7 people & $7 \%$ \\
\hline $18-21$ years old & 50 people & $50 \%$ \\
\hline $22-25$ years old & 36 people & $36 \%$ \\
\hline $26-30$ years old & 4 people & $4 \%$ \\
\hline Older than 31 years & 3 people & $3 \%$ \\
\hline
\end{tabular}

(4) Educational background analysis of the Wushu Sanda coaches. A good trainer should not only have a high level of athletic competition, but also have a certain education level. In a survey of all the coaches, the number of coaches with an educational background inferior to junior college is 4 , accounting for $27 \%$ of the total number; the number of coaches with a junior college educational background is 5 , accounting for $32 \%$ of the total number; the number of coaches that have an undergraduate educational background is 6 , accounting for $41 \%$ of the total number; and there are no coaches that have a graduate degree. Judging from Table.4, undergraduate education accounted for the majority and the coaches basically are highly educated, but for some of the coaches, the education levels still need to be improved.

TABLE 4 EDUCATIONAL BACKGROUND STATISTICS OF THE WUSHU SANDA CLUB COACHES

\begin{tabular}{|c|c|c|}
\hline Educational background & Number & Percentage \\
\hline Inferior to junior college & 4 people & $27 \%$ \\
\hline Junior college & 5 people & $32 \%$ \\
\hline Undergraduate & 6 people & $41 \%$ \\
\hline Graduate & 0 people & $0 \%$ \\
\hline
\end{tabular}

(5) Training frequency and time of the Wushu Sanda practitioners. As can be drawn from Table 5, because the purpose and meaning for each person to learn Sanda is very different, the practitioners are dwindling number, and not many people are able to carry out a long-term training. There are 13 people who have been in training more than 12 months and these trainees are mainly for professional Sanda training.

Numbers of practitioners who have been in training for 1-2 months and 3-6 months are in the majority, accounting for $40 \%$ and $26 \%$, respectively. The training time of these people is less than six months. In general, participants, whose training time does not reach half a year, all belongs to beginners. There are 21 practitioners who have exercised for 7-12 months. They should be just out of the role of the beginners. If they continue with their training, there will be a lot of room for improvement.

TABLE 5 PRACTICE TIME STATISTICS OF THE WUSHU SANDA TRAINEES

\begin{tabular}{|c|c|c|}
\hline Practice time & Number & Percentage \\
\hline 1-2 months & 40 people & $40 \%$ \\
\hline 3-6 months & 26 people & $26 \%$ \\
\hline 7-12 months & 21 people & $21 \%$ \\
\hline Over 12 months & 13 people & $13 \%$ \\
\hline
\end{tabular}

C. Factors that influence the Wushu Sanda practitioners to participate in the practice of Wushu Sanda

There are more males than females among the coaches in Nanjing Wushu Sanda clubs, and the number of male and female coaches are respectively 14 (93\%) and 1 (7\%); 
in the coach team, young coaches account for the majority. The number of 20-30 years old coaches is 10 and account for $67 \%$ of the total number of coaches; the number of coaches more than 30 years old is five and account for $33 \%$. As young coaches are in the majority number, there is still some lack of teaching experience. The longest teaching life among the coaches is more than 4 years, and number of these coaches is 4 , which accounts for $13 \%$; the next is 3-4 years, and the number of these coaches is 3 , which accounts for $20 \%$; then the next is $2-3$ years, and the number of these coaches is 4 , which accounts for $27 \%$; the next is 1-2 years, and the number of these coaches is 6 , which accounts for $40 \%$; generally speaking, the coaches in Wushu Sanda clubs have a relatively short coaching life, and number of coaches with professional standards of coaching is less.

Analyzing from the grade of the coaches' sports level, the number of coaches that have a technical level of 1 is 2 , which accounts for $13 \%$ of the total coaches; the number of coaches that have a technical level of 2 is 8 , which accounts for $54 \%$ of the total coaches; and there are still 5 coaches that the technical level is still under 2, accounting for $33 \%$. The survey shows that four coaches are transformed from the martial arts and Taekwondo coaches, and some coaches do not have the professional level of Sanda.

As the liquidity of the trainees in the day-to-day training, the coaches are required to conduct targeted training, as well as to have a reasonable training schedule. If the technical level of the coaches is not high enough, it will affect the technical upgrading of the trainees and finally result in the trainees' losing interest in Sanda, leading to run off for trainees.

In the day-to-day training, participants liquidity is relatively large, which requires coaches targeted to train, but also have a reasonable training schedule, coaches technical level is not high enough, will affect the technical upgrading of the trainees, final cause students to lose interest in Sanda, resulting in attrition. Coaches are the most important commanders in the process of sports training. They themselves should not only have high techniques, good physical fitness and psychological qualities, but also have the ability for research and innovation, management capabilities, and most importantly have the professionalism. Judging from the training program developed by the coaches, 3 Wushu Sanda Clubs in Nanjing do not install the training program, and the coaches make decisions according to their own thoughts in the teaching process, which lacks of scientificalness.

\section{CONCLUSIONS}

By caring out research and analysis of the survey results, it can be seen that the development of Wushu Sanda in fitness clubs in Nanjing plays a positive role in promoting the sport. As for the participants themselves, they can also enhance physical fitness, hone the will and help self-cultivation. Besides, it contributes to shape perfect physique through Sanda training. In addition,
Sanda can also improve the quality, flexibility and coordination of the body. But attention should be paid on that there are still some problems in the process of Wushu Sanda development in the clubs, which mainly are reflected in the following areas:

(1) Support from the Municipal government for the Wushu Sanda is not big enough, and the inputs of the venues and facilities on Sanda are not enough;

(2) With small-scale operation as the main way, the Sanda clubs often operate independently and company joint way is very rare. Besides, the publicity means are very single;

(3) The teaching model of Sanda clubs is relatively simple and boring, not stimulating effectively on the trainees, and it is difficult to draw the student's interest;

(4) Sanda Club coaches are getting younger and younger, so they are relatively lack of experience. Number of coaches that have professional Sanda experience and quality is much less. What is more noteworthy is that some of coaches do not prepare detailed teaching plans;

(5) Rules and regulations of the Sanda clubs are not quite perfect. Only a few clubs have clear rules and regulations of the club, but the enforcement of the club rules has to be raised. In addition, the operation of the club as a whole lacks scientific management.

\section{REFERENCES}

[1] Ruilin Zhan, "Study on Sustainable Development of the Chinese Wushu Sanda”, Science and Technology Innovation Herald,2008.

[2] Yong Zhou, Minli Qiao, “A Comparative Study of the Difference between Chinese Wushu and Korea Taekwondo on Their Popularizing Model”, Journal of Physical Education Institute of Shanxi Normal University,2008,(09).

[3] Guiju Tian, Fagang Chen, "Innovative Development of Competitive Sanda under the Conditions of Market Economy", China Market,2007,(05).

[4] Lianquan Wu, Wei Zhu, Liangcai Jin, et, al, “The Popularity of Sanda”, Wushu Science,2007,(11).

[5] Dan Liu, "Wuhan City Community Martial Arts Fitness Activity Present Situation Investigation and Research", Wuhan Institute of Physical Education,2007.

[6] Faming Guo, Zhilu Zhang, "The Current Situation and the Thoughts on Reformation of Wushu Sanda of Shanxi Province", Wushu Science,2007,(08).

[7] Yangsheng Chen, "Study and Analysis on the Current Conditions of Teachers in Excellent Wushu Schools", Wushu Science,2005,(11).

[8] Rensong Li, "A Study on the Actuality and Countermeasures of Fujian Wushu School”, Wuhan Institute of Physical Education,2007.

[9] Yuechun Zhao, "The Study on Present Condition and Counterplan of Martial a Arts(Building) School in Shandong Province", Guangxi Normal University,2007.

[10] Bingfeng Wang, "Analysis of Colleges to Establish the Wushu Sanda Club Mode”, Neijiang Science \& Technology,2009,(03).

[11] Bing Zhang, "Application of Mathematical Model of Evacuation for Large Stadium Building. Research Journal of Applied Sciences”, Engineering and Technology, 2013, Vol.5, No.04, pp1432-1440.

[12] Chang Y.T, Pei X.P, Xia Q.H. , "Study on basketball offensive defensive under new rules", Journal of Changsha Railway University (Social Science), 2012, Vol.13, No.1, pp.127-128. 
[13] Chen J. , "Characteristics and problems of college students in a basketball game Defensive Tactics”, Contemporary Sports
Technology,

2011

Vol.1,

No.3,

pp.23-24. 